\title{
Renal and Myocardial Histopathology and Morphometry in Rats with Adenine - Induced Chronic Renal Failure: Influence of Gum Acacia
}

\author{
Badreldin H. Alia Ibrahim Inuwa ${ }^{b}$ Mohamed Al Za'abia. Shadia Al Bahlanic \\ Halima Al Issaeic Aishwarya Ramkumara Thulasi Madanagopala Abedrrahim \\ Nemmar $^{d}$ Denise M. Malheiros ${ }^{\mathrm{e}}$ Roberto Zatz
}

Departments of aPharmacology and Clinical Pharmacy, ${ }^{b} \mathrm{Clinical}$ and Human Anatomy, and ${ }^{\mathrm{c} P a t h o l o g y,}$ College of Medicine \& Health Sciences, Sultan Qaboos University, Al-Khod, Sultanate of Oman; dDepartment of Physiology, College of Medicine and Health Sciences, United Arab Emirates University, Al Ain, UAE; ' Laboratório de Fisiopatologia Renal, São Paulo, Brazil

\section{Key Words}

Gum acacia • Adenine $\cdot$ Renal failure $\cdot$ Heart remodeling • Left ventricle $•$ Rat

\begin{abstract}
Background/Aim: Chronic kidney disease (CKD) is associated with increased occurrence of cardiovascular system dysfunction. Previous studies have revealed a number of alterations in the kidneys and heart during CKD. However, unbiased quantitative studies on these structures in this disease have so far not been addressed. Materials and Methods: We induced CKD in rats by feeding adenine $(0.75 \%$ w/w , four weeks) and using unbiased stereological methods, investigated the effect of the ensuing CKD on the kidneys and left ventricular structure. Since gum acacia (GA) has previously been shown to ameliorate the severity of CKD in humans and rodents, we investigated the effect of giving GA $(15 \%$ w/v in the drinking water concomitantly with adenine) on the kidneys and left ventricular structure using the above model. Results: The CKD was confirmed by standard biochemical indices in plasma and urine and by accumulation of the uremic toxin indoxyl sulfate. Additionally, it increased blood pressure. In rats with CKD absolute volume of left ventricle was significantly increased, and the volume density and absolute volume of myocardial capillaries were decreased, whilst the same parameters of myocardium and interstitial tissue were increased. Renal morphometry demonstrated significant increase in kidney volume and interstitial tissue in adenine- treated rats. Similarly, glomerular Bowman's capsule was significantly thickened. The myocardial and renal changes were significantly mitigated by GA treatment. Conclusions: These results add to our existing knowledge of the pathophysiology of adenine - CKD and provides plausible histopathological and morphometric evidence for the usefulness of GA in CKD.
\end{abstract}




\section{Introduction}

In chronic kidney disease (CKD), the incidence of cardiovascular complications, including hypertension, left ventricle (LV) hypertrophy, both systolic and diastolic dysfunctions, autonomic neuropathy and arrhythmia, is on average about 20 times higher compared with the normal population [1,2]. Cardiovascular diseases (mainly heart failure, ischemic heart disease or sudden cardiac death) are the leading causes of death in CKD patients [2]. Both structural and functional changes in the heart have been described due to experimental CKD [3].

Some pathological processes are known to affect the left ventricular function. For example the impact of experimental diabetic cardiomyopathy on electrophysiological characteristics of the rat heart or the negative effect on the contractility of the end-stage heart failure in spontaneous hypertensive rats are more expressed in the LV $[3,4]$. With regards to $\mathrm{CKD}$, the majority of the experimental studies were focused on LV function, while only minimal attention was paid to quantitative structural studies. An unbiased stereological study on the LV in CKD models is, to the best of our knowledge, so far lacking.

Ali et al. [5] have recently demonstrated that gum acacia (GA) treatment was effective in ameliorating several biochemical, physiological and histopathological indices, and motor and behavioral changes in adenine-induced CRF [6]. However, the effect of adenine-induced CKD on cardiac microstructure in rats, and the possible protective effects of GA thereon have not been investigated.

Therefore, in this study, we report on an unbiased stereological approach to quantify the myocardial structural changes that occur in adenine-induced CKD in rats and whether treatment with GA would mitigate these changes.

\section{Materials and Methods}

\section{Animal experiments}

Male Wistar rats (9-10 weeks old, weighing $270 \mathrm{~g}$ in average) were housed in a room at a temperature of $22-23^{\circ} \mathrm{C}$, relative humidity of about $60 \%$, with a $12 \mathrm{~h}$ light-dark cycle (lights on at 6:00), and free access to standard pellet chow diet containing $0.85 \%$ phosphorus, $1.12 \%$ calcium, $0.35 \%$ magnesium, $25.3 \%$ crude protein and $2.5 \mathrm{IU} / \mathrm{g}$ vitamin D3 (Oman Flour Mills, Muscat, Oman) and water. The study was approved by the University Animal Ethical Committee and was conducted in conformity with international laws and policies (EEC Council directives 86 / 609, OJL 358, 1 December, 12, 1987; NIH Guide for the Care and Use of Laboratory Animals, NIH Publications No. 85-23, 1985).

\section{Experimental Design}

After an acclimatization period of one week, rats $(n=24)$ were randomly divided into four equal groups and treated for four consecutive weeks. The first group continued to receive the same diet without treatment until the end of the study (control group). The second group was switched to a powder diet containing Adenine (0.75\% w/w in feed). The third group was given normal food and GA (Supergum EM 10) in drinking water at a concentration of $15 \% \mathrm{w} / \mathrm{v}$. The fourth group was given adenine in the feed as in group two, plus GA in drinking water at a concentration of $15 \%$ w/v. The dose of adenine was chosen from the original method by Yokozawa et al. [7] and the dose of GA was chosen as was reported earlier [8]. During the treatment period, the rats were weighed weekly. For the collection of urine, they were placed individually in metabolic cages for $24 \mathrm{~h}$, after the 28 days treatment period. On the morning after the metabolic sampling, the rats were anesthetized with an intraperitoneal injection of ketamine (75 mg/kg) and xylazine (5 mg/ $\mathrm{kg}$ ), and blood (about $3.5 \mathrm{~mL}$ ) was collected from the anterior vena cava and placed into plain or heparinized tubes. The blood and urine were centrifuged at $900 \mathrm{~g}$ at $4^{\circ} \mathrm{C}$ for $15 \mathrm{~min}$. The serum or plasma obtained, together with the urine specimens, was stored at $-80^{\circ} \mathrm{C}$ to await analysis within 4 weeks after the end of the treatment. 


\section{Cellular Physiology $\quad$ Cell Physiol Biochem 2014;34:818-828

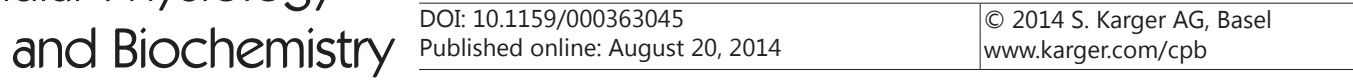 \\ Ali et al.: Renal and Myocardial Histopathology and Morphometry in Chronic Renal Failure}

Biochemical Methods

The concentrations of urea, creatinine, protein, calcium and phosphorus in plasma, serum and/ or urine were estimated spectrophotometrically using commercial kits (Human GmbH, Mannheim, Germany). Creatinine clearance (CCr) was calculated as reported before [9]. Plasma nitrite/nitrate concentrations were measured by an ELISA method using a commercial kit (R\&D, Oxon, UK). Plasma indoxyl sulfate concentration was measured by an HPLC method [10].

\section{Perfusion fixation and tissue sampling}

After induction of anesthesia, the abdomen was opened via a midline incision. The inferior vena cava and abdominal aorta were cannulated. Before perfusion fixation, anticoagulant sodium heparin (1000 units) was injected through the inferior vena cava, and followed one minute later by a bolus injection of 1.5 $\mathrm{mL} 2 \%$ procaine in physiological saline to arrest the heart in diastole and abolish vascular tone. The heart

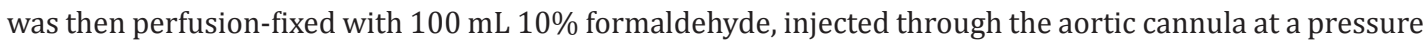
of $160 \mathrm{~mm}$ Hg. Thereafter, the kidneys were removed and place in 10\% neutral-buffered formalin pending analysis. The heart was excised, and the LV free wall plus septum was dissected out for the determination of its mass (LVM) and absolute volume $\left(\mathrm{LVV}_{\mathrm{A}}\right)$, and for tissue sampling.

The tissues were dehydrated in increasing concentrations of ethanol, cleared with xylene and embedded in paraffin. Three micrometer sections were prepared from renal and myocardial paraffin blocks and stained with hematoxylin and eosin (H \& E), periodic acid-Schiff (PAS) and Masson's trichrome stains. The slides were evaluated by a histopathologist in a blinded fashion.

\section{Stereological analysis}

After weighing, the $\mathrm{LVV}_{\mathrm{A}}$ was determined using the Cavalieri method [11]. In brief, a series of parallel sections were cut through the LV or the whole kidney at fixed thicknesses (T). This generated a series of slabs, which were all laid the same way up. Traditional point counting was used to estimate the area (A) of each of the cut surfaces of all slabs. Absolute volume, $\mathrm{LVV}_{\mathrm{A}}$ was then calculated by the equation:

$\mathrm{LVV}_{\mathrm{A}}=\mathrm{A} \times \mathrm{T}$

Tissue sampling was performed according to the orientator method [12]. Briefly, a slab was randomly selected from those used for evaluating $\mathrm{V}_{\mathrm{A}^{\prime}}$ and processed via a series of cuts accordingly [13]. The last cut surface was considered to be uniformly isotropic. One block of tissue was made from each ventricle, and was embedded in paraffin wax. Using a Leica microtome (Leica RM2165; Heerberg, Switzerland), a threemicron-thick section was obtained from each block and were stained as described above. Two random sections were obtained from each block of tissue. A systematic uniform random sample of six fields was obtained from each section and was digitally stored using a camera attached to an Olympus microscope (BH2; Olympus Optical Co, Tokyo, Japan).

Stereological measurements of volume density $\left(\mathrm{V}_{\mathrm{D}}\right)$ measurements of left ventricle components (capillaries $\mathrm{V}_{\mathrm{DC}}$, muscle $\mathrm{V}_{\mathrm{DM}}$, and interstitium $\mathrm{V}_{\mathrm{DI}}$ ), kidney components (interstitium and nephron) and thickness of glomerular Bowman's capsule, $\mathrm{BC}_{\mathrm{T}}$ were performed with stereology software (Histometrix, MIL 6 Version 1; Kinetic Imaging Ltd, Nottingham, UK) installed on a personal computer. Only micro-vessels without an apparent smooth muscle wall in the heart were included. Absolute volumes of capillaries $\left(\mathrm{V}_{\mathrm{AC}}\right)$, myocardium $\left(\mathrm{V}_{\mathrm{AM}}\right)$ and interstitial tissue $\left(\mathrm{V}_{\mathrm{AI}}\right)$ in left ventricle were calculated using the mathematical equation;

$$
\mathrm{V}_{\mathrm{A}(\mathrm{C}, \mathrm{M}, \mathrm{l})}=\mathrm{V}_{\mathrm{D}(\mathrm{C}, \mathrm{M}, \mathrm{l})} \times \mathrm{LVV}_{\mathrm{A}}
$$

\section{Statistical analysis}

Data were expressed as means \pm SEM, and were analyzed with GraphPad Prism Version 4.01 for Windows software (Graphpad Software Inc., San Diego, USA). Comparisons between the four groups were performed by analysis of variance ANOVA, followed by Newman-Keuls multiple-range tests. $P$ values $<0.05$ are considered significant. 
Table 1. Effect of Gum Acacia (GA, 15\% w/v 4 weeks) on some physiological parameters in rats treated with adenine $(0.75 \%$ w/w 4 weeks). Values in the table are mean \pm SEM $(n=6)$. Adenine was added to the feed at a concentration of $0.75 \%$ w/w for 4 weeks, and gum Acacia (GA) was given at a concentration of $15 \%{ }^{w / v}$ for 4 weeks. ${ }^{*} P<0.05$, compared to Control group; ${ }^{\#} P<0.05$, compared to Adenine-treated group

\begin{tabular}{lcccc}
\hline Parameters/Group & Control & Adenine & GA & Adenine+GA \\
\hline Body weight change \% & $31.13 \pm 3.83$ & $44.52 \pm 2.73^{*}$ & $5.76 \pm 3.79^{*}$ & $34.55 \pm 2.04$ \\
Kidney weight (\% body weight) & $0.85 \pm 0.02$ & $3.60 \pm 0.07^{*}$ & $0.91 \pm 0.02$ & $1.41 \pm 0.12^{\#}$ \\
Water intake (ml/day) & $16.83 \pm 2.18$ & $36.80 \pm 5.02^{*}$ & $18.00 \pm 3.26$ & $27.00 \pm 1.71$ \\
Urine output (ml/day) & $7.50 \pm 0.88$ & $31.16 \pm 2.16^{*}$ & $5.83 \pm 0.74$ & $16.33 \pm 1.11^{\#}$ \\
\hline
\end{tabular}

Table 2. Effect of Gum Acacia (GA, $15 \%$ w/v 4 weeks) on blood pressure and heart rate in rats treated with adenine $\left(0.75 \%{ }^{\text {w/w }} 4\right.$ weeks). Values in the table are mean \pm SEM $(n=6)$. Adenine was added to the feed at a concentration of $0.75 \%{ }^{\mathrm{w} / \mathrm{w}}$ for 4 weeks, and gum acacia (GA) was given at a concentration of $15 \%{ }^{\text {w/v }}$ for 4 weeks. ${ }^{*} P<0.05$, compared to Control group; ${ }^{\#} P<0.05$, compared to Adenine-treated group

\begin{tabular}{lllll}
\hline Parameters/Group & Control & Adenine & GA & Adenine + GA \\
\hline Systolic blood pressure (mm Hg) & $127 \pm 4.77$ & $164 \pm 6.03^{*}$ & $129 \pm 4.96$ & $133 \pm 5.17^{\#}$ \\
Diastolic blood pressure (mm Hg) & $69 \pm 4.65$ & $115 \pm 8.02^{*}$ & $64 \pm 2.46$ & $68 \pm 2.16^{\#}$ \\
Heart rate (BPM) & $360 \pm 9.97$ & $325 \pm 20.41$ & $358 \pm 11.27$ & $348 \pm 13.78^{\#}$ \\
\hline
\end{tabular}

Table 3. Effect of Gum Acacia (GA, 15\% w/v 4 weeks) on some biochemical parameters in rats treated with adenine $\left(0.75 \%{ }^{\text {w/w }} 4\right.$ weeks). Values in the table are mean \pm SEM $(n=6)$. Adenine was added to the feed at a concentration of $0.75 \%{ }^{w / w}$ for 4 weeks, and gum Acacia (GA) was given at a concentration of $15 \%{ }^{\text {w/v }}$ for 4 weeks. ${ }^{*} P<0.05$, compared to Control group; ${ }^{\#} P<0.05$, compared to Adenine-treated group

\begin{tabular}{lcccc}
\hline Parameters/Group & Control & Adenine & GA & Adenine + GA \\
\hline Plasma urea $(\mu \mathrm{mol} / \mathrm{l})$ & $6.36 \pm 0.80$ & $150.71 \pm 2.89^{*}$ & $13.97 \pm 1.23$ & $26.14 \pm 0.98^{\#}$ \\
Plasma creatinine $(\mu \mathrm{mol} / \mathrm{l})$ & $63.09 \pm 4.76$ & $205.57 \pm 12.45^{*}$ & $54.53 \pm 3.02$ & $89.71 \pm 11.31^{\#}$ \\
Creatinine clearance $(\mathrm{ml} / \mathrm{min})$ & $1.06 \pm 0.20$ & $0.29 \pm 0.04^{*}$ & $0.94 \pm 0.19$ & $0.55 \pm 0.06$ \\
Urinary protein $(\mathrm{g} / \mathrm{l})$ & $18.4 \pm 1.94$ & $98.25 \pm 5.77^{*}$ & $23.02 \pm 2.50$ & $57.65 \pm 5.47^{\#}$ \\
Plasma Calcium $(\mathrm{mmol} / \mathrm{l})$ & $3.18 \pm 0.14$ & $1.52 \pm 0.24^{*}$ & $2.87 \pm 0.16$ & $2.9 \pm 0.21^{\#}$ \\
Serum Phosphorus $(\mathrm{mg} / \mathrm{dl})$ & $14.35 \pm 5.86$ & $30.73 \pm 12.54^{*}$ & $15.47 \pm 6.31$ & $8.40 \pm 3.43^{\#}$ \\
Plasma nitrate $(\mu \mathrm{mol} / \mathrm{l})$ & $55.79 \pm 26.02$ & $284.22 \pm 44.22^{*}$ & $54.60 \pm 16.75$ & $222.19 \pm 38.90$ \\
Plasma nitrite $(\mu \mathrm{mol} / \mathrm{l})$ & $60.27 \pm 14.77$ & $230.42 \pm 38.28^{*}$ & $36.64 \pm 2.50$ & $113.45 \pm 18.30^{\#}$ \\
Indoxyl sulfate $(\mu \mathrm{mol})$ & $1.79 \pm 0.81$ & $159.55 \pm 13.46^{*}$ & $0.00 \pm 0.00$ & $1.48 \pm 0.70^{\#}$ \\
\hline
\end{tabular}

\section{Results}

Physiological effects

Table 1 shows the changes in body weights, relative kidney weights, as well as water intake and urine output in the four experimental groups. Adenine feeding alone significantly decreased body weight by about $44 \%$ when compared with the initial body weight. Rats 


\begin{tabular}{|c|c|c|}
\hline Cellular Physiology & Cell Physiol Biochem 2014;34:818-828 & \\
\hline and Biochemistry & $\begin{array}{l}\text { DoI: 10.1159/000363045 } \\
\text { Publisnea online: August 20, } 2014\end{array}$ & $\begin{array}{l}\text { O } 2014 \text { S. Karger AG, Basel } \\
\text { www.karger.com/cpb }\end{array}$ \\
\hline
\end{tabular}

Fig 1. A representative micrograph showing kidney histomorphology in control rat (A) and rats treated for 4 weeks with adenine $\left(0.75 \%{ }^{\mathrm{w} / \mathrm{w}}\right.$ in the feed) (B), gum acacia (GA, $15 \%$ w/v in drinking water) (C) or adenine + GA simultaneously) (D), using H \& E (a), Trichrome Masson (b) and PAS (c) stains. No changes were seen in either untreated or gum acacia-treated controls (A and $\mathrm{C}$ ). Marked expansion and hypercellularity of the interstitial area were noted in adenine-treated rats, along with increased amounts of collagenlike material (B). These abnormalities were markedly attenuated in adeninetreated rats that also received gum acacia (D). No glomerular or vascular injury was detected in any of the groups. Bowman's capsule (arrows), polymorphonuclear cell nuclei (arrowhead) are shown.

treated with adenine alone drank significantly more water and voided more urine compared to the control group, and the relative kidney weight in the former group was increased three-fold. These effects were significantly reversed with the addition of GA to the adenine- treated rats.

The data concerning SBP, DBP and HR at the end of the 4-week experimental protocol are presented in Table 2. Diastolic BP showed a significant increase in adenine - treated group when compared with the control or GA groups. $(P<0.05)$. GA treatment has no significant effect on either the SBP or the DBP when to the corresponding values in the controls. In rats given adenine and GA, there was a significant reduction in both SBP and DBP when compared to the adenine
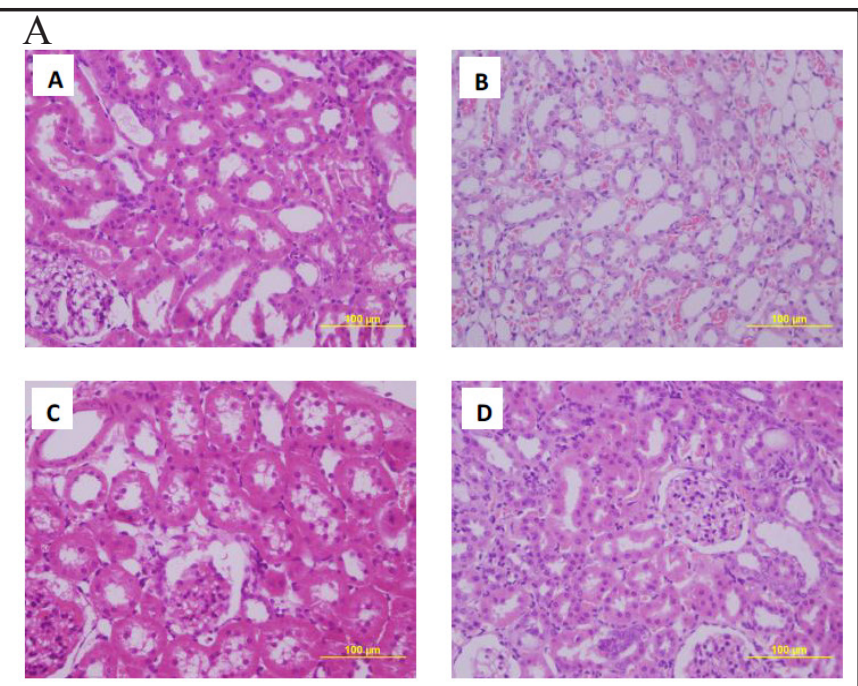

\section{B}
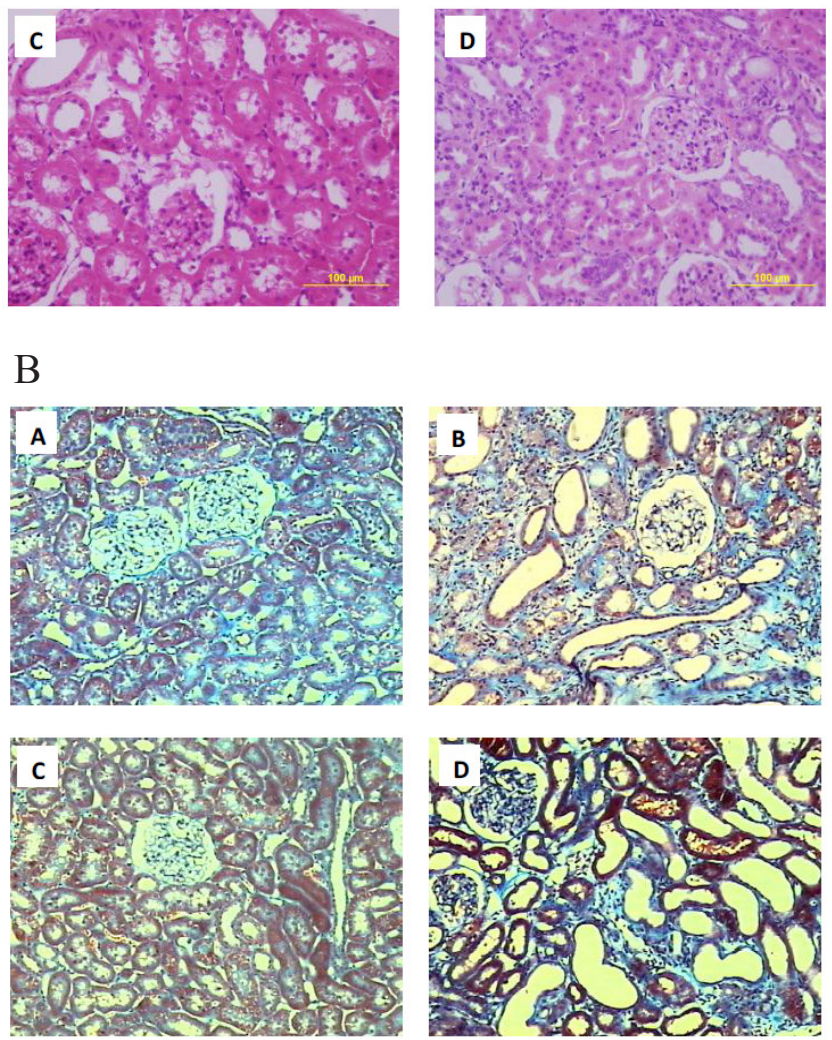

C
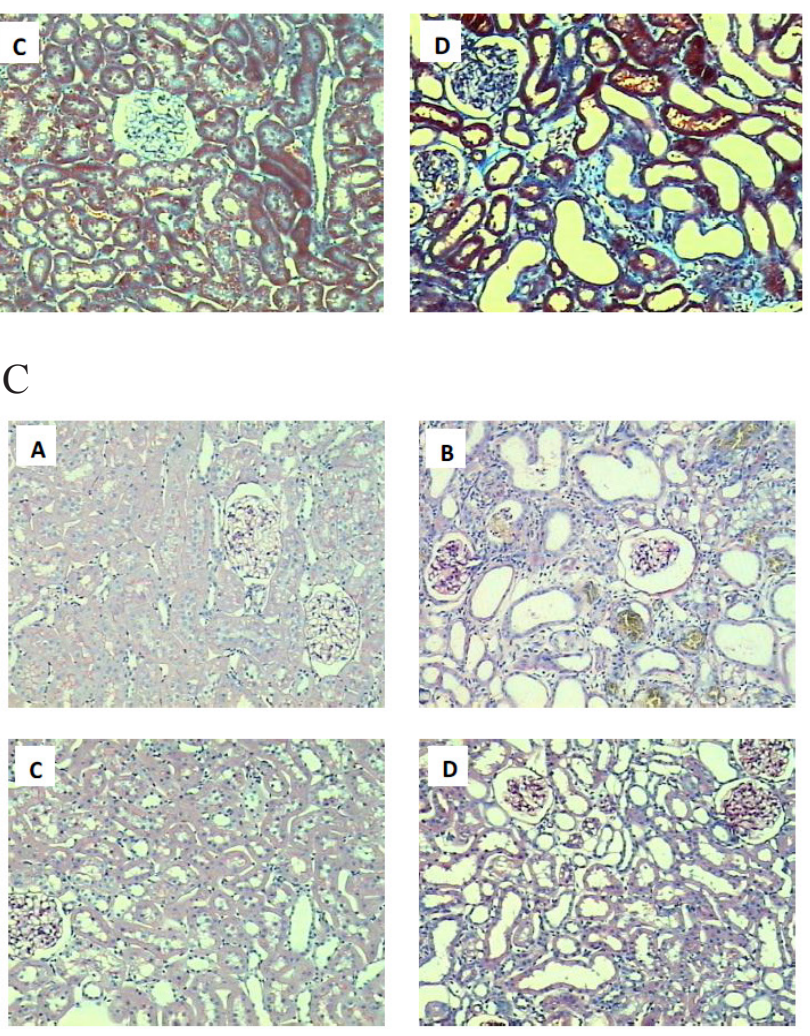

-treated group. There was no significant difference in the heart rates between all four experimental groups. 


\section{Cellular Physiology Cell Physiol Biochem 2014;34:818-828

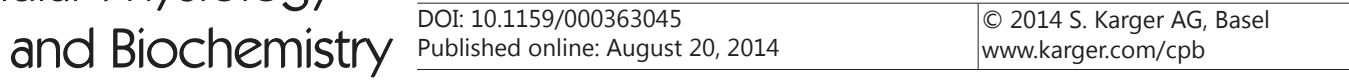 \\ Ali et al.: Renal and Myocardial Histopathology and Morphometry in Chronic Renal Failure}

Fig 2. A representative micrograph showing left ventricle histomorphology in control rat $(\mathrm{A})$ and rats treated for 4 weeks with adenine $(0.75 \%$ w/w in the feed) (B), gum acacia (GA, 15\% $w / v$ in drinking water) (C) or adenine + GA simultaneously) (D), using $\mathrm{H}$ $\&$ E and PAS stains. Capillary (black arrow), fibrosis (white arrow) are shown.

Fig 3. cortical IN thickness (INT) in control rat (A) and rats treated for 4 weeks with adenine $(0.75 \%$ w/w in the feed) (B), gum acacia (GA, 15\% ${ }^{\mathrm{w} / \mathrm{v}}$ in drinking water) (C) or adenine $+\mathrm{GA}$ simultaneously) (D).
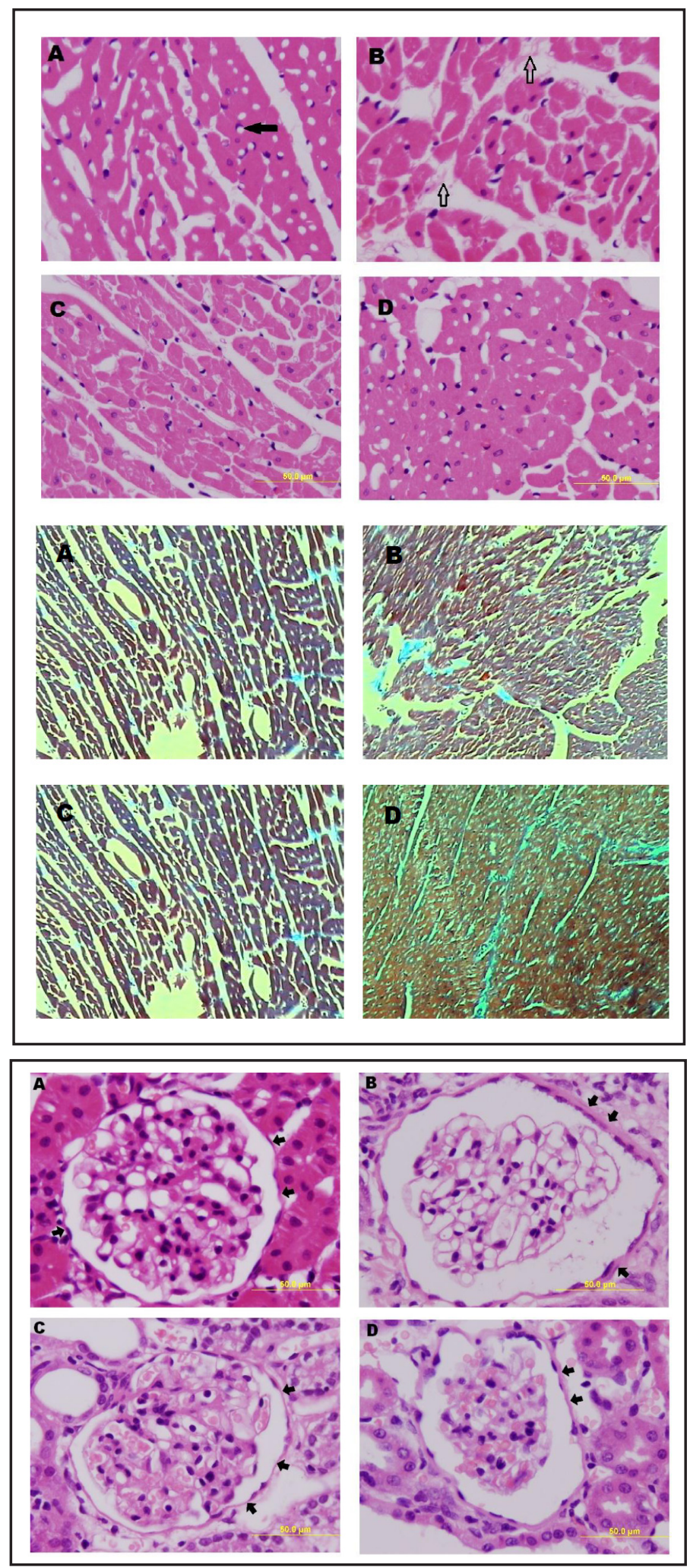

Biochemical measurements

Table 3 shows the biochemical parameters in each of the four experimental groups. Adenine feeding $\left(0.75 \%{ }^{\mathrm{w} / \mathrm{w}}\right.$ for 4 weeks $)$ caused significant increases $(P<0.05)$ in the concentrations of plasma urea and creatinine, and a significant decrease in the creatinine 
Fig 4. Absolute volumes (in $\mu \mathrm{m}^{3}$ ) of kidneys (A), and of left Kidney components $(\mathrm{B} \& \mathrm{C})$ in control rats and rats treated for 4 weeks with adenine $\left(0.75 \%{ }^{w / w}\right.$ in the feed), gum acacia (GA, 15\% w/v in drinking water) or adenine + GA simultaneously). Each column and vertical bar represent mean \pm SEM $(n=6) . P<0.05$ was considered significant.

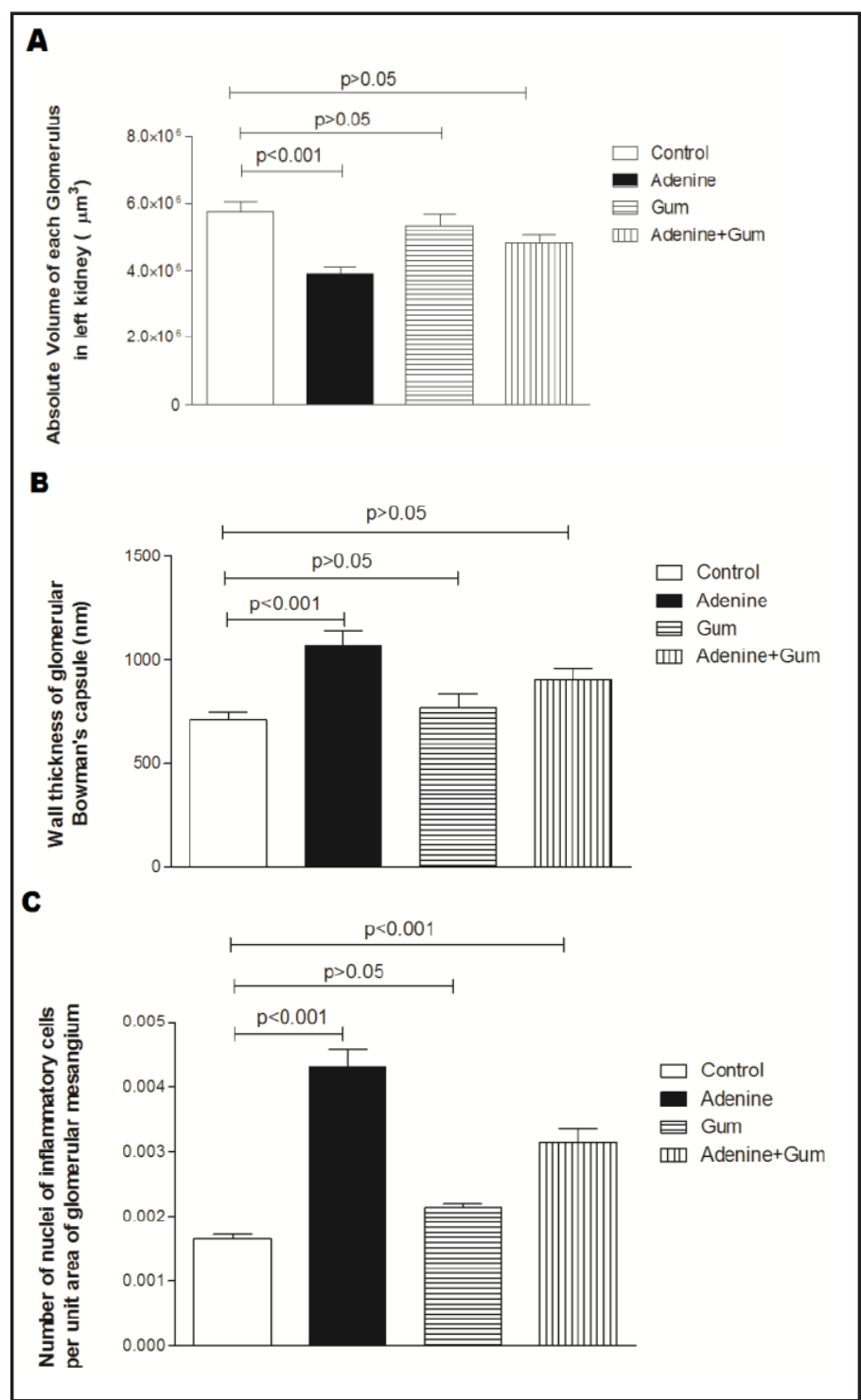

clearance $(P<0.05)$ compared to control group. As a further marker of kidney injury and established uremia, proteinuria and plasma concentration of indoxyl sulfate (a major uremic toxin) were analyzed showing a significant increase of urinary protein and plasma indoxyl sulfate in adenine-treated rats compared to controls. Most of these biochemical changes found in the adenine - treated rats were significantly abated in rats treated with adenine plus GA. There was no significant difference in any of the biochemical parameters measured between control rats and rats treated with GA alone.

\section{Histopathological changes}

Histopathological features of the kidney parenchyma stained with Hematoxylin and eosin (H\&E), Trichrome Masson and PAS are illustrated in Figures $1 \mathrm{a}, \mathrm{b}$ and c, respectively.

No changes were seen in either untreated or gum acacia-treated rats (A and C, respectively). Marked expansion and hypercellularity of the interstitial area were noted in adenine-treated rats, along with increased amounts of collagen-like material (B). These abnormalities were markedly attenuated in adenine-treated rats that also received gum acacia (D). No glomerular or vascular injury was detected in any of the groups.

Histopathological features of the left ventricle myocardium stained with H\&E and PAS are illustrated in Figure 2. Control rats and rats treated with GA only (A and C, respectively) 
Ali et al.: Renal and Myocardial Histopathology and Morphometry in Chronic Renal Failure

Fig 5. Absolute volumes (in $\mu \mathrm{m}^{3}$ ) of left ventricle (A) and its components: muscle, (A) interstitium (B) capillaries (C) in control rats and rats treated for 4 weeks with adenine $0.75 \% \mathrm{w} / \mathrm{w}$ in the feed), gum acacia (GA, 15\% w/v in drinking water) or adenine $+\mathrm{GA}$ simultaneously). Each column and vertical bar represent mean \pm SEM (n=6). $P<0.05$ was considered significant.

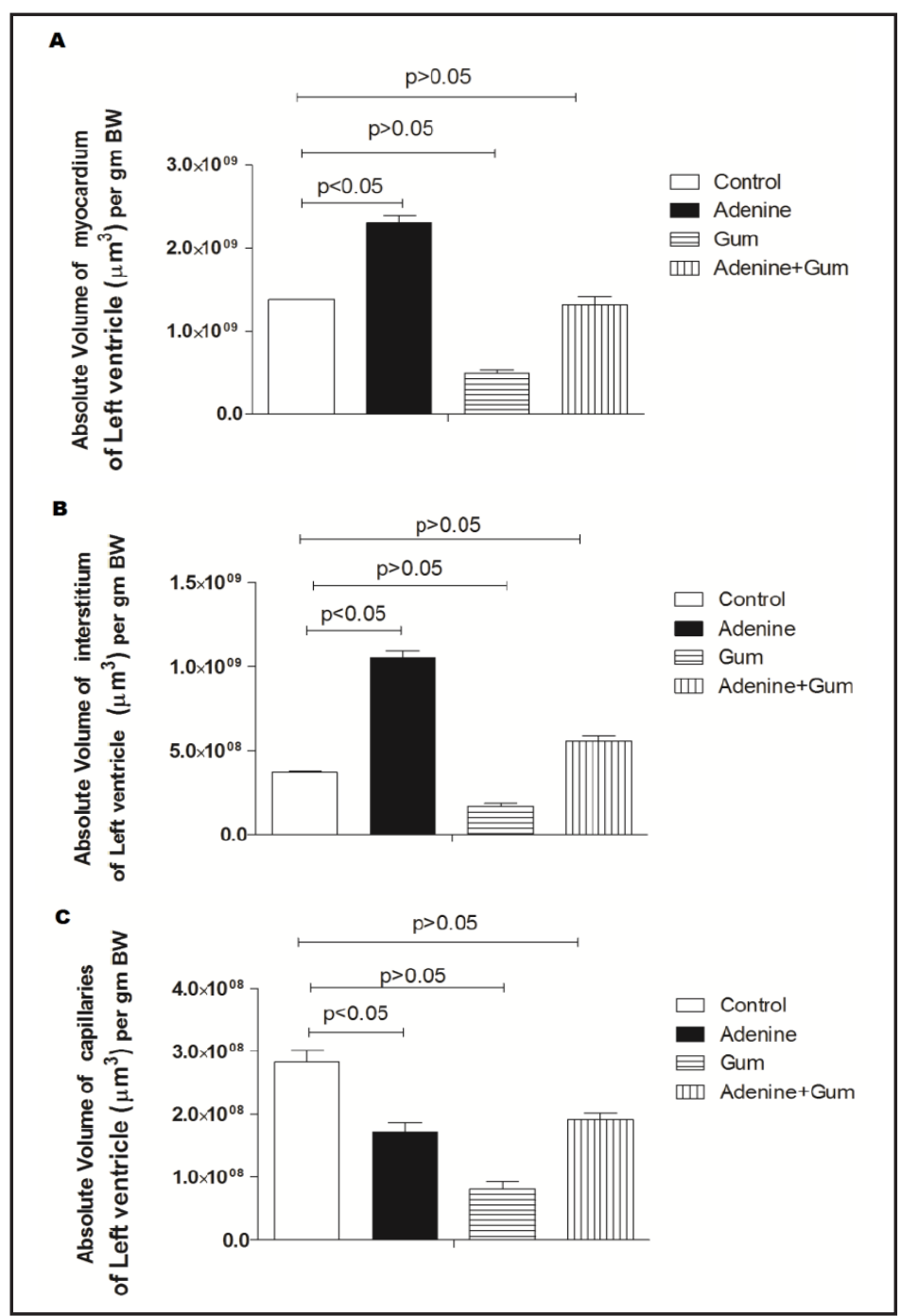

showed normal myocardium histology. The only obvious qualitative feature in adeninetreated rats was the presence of diffuse fibrosis and myocardial hypertrophy as compared to control rats.

\section{Stereological changes}

Quantitative morphometric kidney data are illustrated in Figures 3 and 4. Mean absolute volume of left kidney was doubled in adenine - treated rats as compared to control group. This increase was revered in rats treated with adenine + GA. There was no significant difference in left kidney volume between control and GA -treated rats. Analysis of the cortical IN thickness (INT) showed that it was significantly increased in adenine -treated rats when compared to the controls and the GA -treated rats. The action was reversed but not completely when GA was given to the rats that received adenine (Fig 3). The increase in kidney volume is illustrated in interstitial fibrous tissue accounted for most of the increase in kidney volume in adenine - treated rats with volume of glomeruli and tubules actually decreased. These changes were significantly reversed with addition of GA to adenine treated rats.

Quantitative myocardial data are illustrated in Figure 5. The mean absolute volume of the left ventricle was significantly increased in adenine - treated rats. This increase was mainly contributed by the myocardium and interstitial tissue with the capillary bed actually decreased. These changes were not observed in rats treated with adenine plus GA. 


\section{Discussion}

In this work we reported on the structural and morphometric alterations in the renal and myocardial tissues that occur subsequent to adenine -induced CKD. The changes in the renal structure and in the vascular function in rats with adenine - induced CKD have recently been investigated, $[8,9,14,15]$ but as far as we are aware, there are no reports on similar morphometric analysis of the myocardium in this animal model.

Adenine feeding $\left(0.75 \%{ }^{\mathrm{w} / \mathrm{w}}\right.$ for four weeks) induced CKD as judged by the histopathological alterations in the kidneys, and by the significant increases in water intake and urine production, and in proteinurea, accumulation of the uremic toxin indoxyl sulfate, creatinine and urea concentrations, and the decrease in creatinine clearance, as well as in body weight. As a consequence of the adenine - induced CRF, blood pressure was significantly raised, and this has been recently reported [16]. Also, in confirmation of an earlier work, [6, 9] the co-administration of the natural product GA was effective in ameliorating all the above adenine-induced changes.

Our results on renal morphology further support earlier findings demonstrating glomerulosclerosis in adenine induced CKD [9]. Previous studies on adenine-induced renal changes have been qualitative in nature [5]. In this paper, we provide innovative quantitative data that supports above previous observations. Thickness of Bowman's capsule is linked to the deposition of fibrous tissue usually secondary to chronic inflammation. Glomerular messangial tissue cellularity further indicates infiltration with inflammatory cells thereby linking it with later development of glomerulosclerosis. The functional correlates of these quantitative changes include the deterioration in creatinine clearance, the significant increases in water intake and urine production, and the increases in creatinine and urea concentrations observed in adenine- CKD. Accumulation of the uremic toxin (as was shown here) is known to cause, among other actions, several structural changes in the kidneys such as inhibition of tubular epithelial cell proliferation, probably mediated by stress in the endoplasmic reticulum.

The proposed mechanism by which GA may have produced a salutary action on the kidney has been discussed earlier $[8,17,18,19]$. Briefly, GA exerts both anti-inflammatory and anti-oxidant actions, and these may counteract the generation of free radicals and the inflammation that - is associated with adenine -induced CKD. Also, we have recently shown that adenine-treated rats showed by Western blotting a detectable caspase- 3 cleavage, which may be considered a possible mechanism involved in the adenine-induced CKD. The induced apoptosis was almost totally suppressed by GA in rat kidney tissue [5]. Recently, it has been reported that the complex inflammatory phenomena associated with adenine-induced CKD

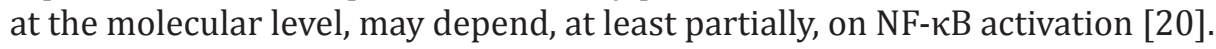

We have shown in a previous similar work, [14] adenine -induced CKD causes a significant rise in systolic and diastolic blood pressure, with variable and insignificant effect on heart rate. GA was effective in ameliorating these actions. The possible mechanisms for this finding have been discussed [15]. Similarly, structural remodeling of the heart characterized by left ventricular hypertrophy (LVH) and expansion of interstitial tissue has been reported in uremic patients [21] and rats with partial renal ablation [22]. In the latter model, an antioxidant ( $\mathrm{dl}-\alpha$ - tocopherol) has been found to abrogate the renal insult. In this study we present quantitative morphometric characteristics of the LVH supporting the observed physiologic changes and that addition of GA has significantly reversed those changes.

As far as we are aware, there is no previously published work on the effect of GA on the heart in CKD. However, a single report by has shown that GA protects against doxorubicin cardiotoxicity in rats [23]. Some of the possible mechanism by which GA reverses the remodeling of the LV could involve its effects on the metabolism of some minerals such as phosphate and/or its antioxidant action.

Disturbances of the mineral metabolism are early and frequent occurrence in CKD [24]. The progressive loss of renal function leads to the lower production of calcitriol and phosphorus retention leading to hypocalcemia, as well as hyperphosphatamia. Moreover, 
recent studies have shown the association of hyperrphosphatemia with LVH as well as with increased mortality in CKD patients [25]. It could well be that the beneficial effects of GA in reducing $\mathrm{LVH}$ and fibrosis is linked to it action in improving renal handling of serum calcium and phosphate. In fact, we did find that adenine -induced CRF causes hyperphosphatemia and hypocalcaemia.

Oxidative stress has been established to be a constant feature in CKD, and may be the basis of the resulting hypertension and other consequences [26]. Several authors have reported that cardiac remodeling characterized by LVH and coronary atherosclerosis is seen in uremic patients [27]. GA has been shown in previous studies to cause a decrease in the concentrations of several pro-inflammatory cytokines in plasma and kidneys of rats suffering from adenine-induced CRF [8]. Similarly, GA has been shown to decrease adenine-induced superoxide production and DNA damage in CKD model [8]. These anti-inflammatory and antioxidant capacities of GA may therefore add to the explanation of its effects in reversing the myocardial changes.

In conclusion, this study presents renal and myocardial morphometric data that supported the observed physiologic changes and correlated well with documented renal and cardiac remodeling seen in CRF. We add in this paper a further structural characterization of this remodeling to be myocardial hypertrophy and interstitial fibrosis, as well as myocardial capillary reduction.

\section{Acknowledgements}

This work was supported by a grant from the Research Council of Oman (RC/Med/ Pharm/10/01). Thanks are due to the staff of the SQU Small Animal House for looking after the animals. GA was a kind gift from Sanwa-Cho, Toyonaka, Osaka, Japan

\section{References}

1 Kundhal K, Lok CE: Clinical epidemiology of cardiovascular disease in chronic kidney disease. Nephron Clin Pract 2005;101:47-52.

- 2 Foster MC, Rawlings AM, Marrett E, Neff D, Willis K, Inker LA, Coresh J, Selvin E: Cardiovascular risk factor burden, treatment, and control among adults with chronic kidney disease in the United States. Am Heart J 2013;166:150-156.

3 Svíglerová J, Kuncová J, Nalos L, Holas J, Tonar Z, Rajdl D, Stengl M: Cardiac remodeling in rats with renal failure shows interventricular differences. Exp Biol Med (Maywood) 2012;237:1056-1067.

4 Casis 0, Gallego M, Iriarte M, Sánchez-Capula JA: Effects of diabetic cardiomyopathy on regional electrophysiologic characteristic of rat ventricle. Diabetologia 2000;43:101-109.

-5 Ali BH, Al-Salam S, Al Za'abi M, Waly MI, Ramkumar A, Beegam S, Al-Lawati I, Adham SA, Nemmar A: New model for adenine-induced chronic renal failure in mice, and the effect of gum acacia treatment thereon: Comparison with rats. J Pharmacol Toxicol Methods 2013;68:384-393.

6 Ali BH, Ziada A, Al Husseni I, Beegam S, Nemmar A: Motor and behavioral changes in rats with adenineinduced chronic renal failure: influence of acacia gum treatment. Exp Biol Med (Maywood) 2011;236:107112.

-7 Yokozawa T, Zheng PD, Oura H, Koizumi F. Animal model of adenine-induced chronic renal failure in rats: Nephron 1986; 44:230-234.

8 Ali BH, Al-Husseni I, Beegam S, Al-Shukaili A, Nemmar A, Schierling S, Queisser N, Schupp N: Effect of gum arabic on oxidative stress and inflammationin adenine-induced chronic renal failure in rats. PLoS One 2013;8:e55242

9 Ali BH, Al-Salam S, Al Husseni I, Kayed RR, Al-Masroori N, Al-Harthi T, Al Zaabi M, Nemmar A: Effects of Gum Arabic in rats with adenine-induced chronic renal failure. Exp Biol Med (Maywood) 2010;235:373382. 


\section{Cellular Physiology Cell Physiol Biochem 2014;34:818-828

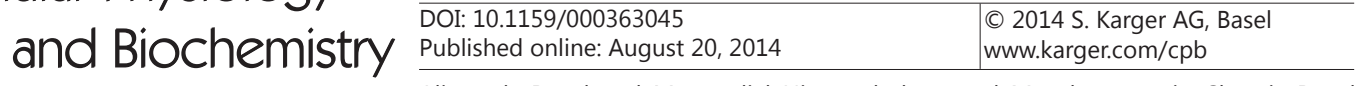 \\ Ali et al.: Renal and Myocardial Histopathology and Morphometry in Chronic Renal
Failure}

10 Al Za'abi M, Ali BH, Al Toubi M: HPLC-Fluorescence method for measurement of the uremic toxin indoxyl sulfate in plasma. J Chromatogr Sci 2013;51:40-43.

11 Amann K, Wiest G, Zimmer G: Reduced capillary density in the myocardium of uremic rats - A stereological study. Kidney Int 1992;42:1079-1085.

12 Mattfeldt T, Mall G, Gharehbaghi H, Moller P: Estimation of surface area and length with the orientator. J Microsc 1990;159:301-317.

13 Howard CV, Reed MG: Estimation of Component Volume and Volume Fraction in Unbiased Stereology. Three-Dimensional Measurement in Microscopy. Oxford: Bios Scientific Publishers Ltd, 1998, pp 55-65.

- 14 Ali BH, Ziada A, Al Husseni I, Beegam S, Al-Ruqaishi B, Nemmar A: Effect of Acacia gum on blood pressure in rats with adenine-induced chronic renal failure. Phytomedicine 2011;18:1176-1180.

15 Nguy L, Johansson ME, Grimberg E, Lundgren J, Teerlink T, Carlström M, Lundberg JO, Nilsson H, Guron G: Rats with adenine-induced chronic renal failure develop low-renin, salt-sensitive hypertension and increased aortic stiffness. Am J Physiol Regul Integr Comp Physiol 2013;304:R744-752.

16 Nguy L, Nilsson H, Lundgren J, Johansson ME, Teerlink T, Scheffer PG, Guron G: Vascular function in rats with adenine-induced chronic renal failure. Am J Physiol Regul Integr Comp Physiol 2012;302:R14261435.

17 Ali BH, Ziada A, Blunden G: Biological effects of gum arabic: a review of some recent research. Food Chem Toxicol 2009;47:1-8.

18 Nasir O. Renal and extrarenal effects of Gum Arabic (Acacia Senegal) - what can be learned from animal experiments? Kidney Blood Press Res 2013;37:269-279.

19 Nasir O, Umbach AT, Rexhepaj R, Ackermann TF, Bhandaru M, Ebrahim A, Artunc F, Kempe DS, Puchchakayala G, Siraskar B, Föller M, Saeed A, Lang F. Effects of gum arabic (Acacia senegal) on renal function in diabetic mice. Kidney Blood PressRes 2012;35:365-372.

-20 Okabe C, Borges RL, de Almeida DC, Fanelli C, Barlette GP, Machado FG, Arias SC, Malheiros DM, Camara NO, Zatz R, Fujihara CK: NF- $\kappa$ B activation mediatescrystal translocation and interstitial inflammation in adenine overload nephropathy. Am J Physiol Renal Physiol 2013;305:F155-163.

21 Amann K, Breitbach M, Ritz E, Mall G: Myocyte/capillary mismatch in the heart of uremic patients. J Am Soc Nephrol 1998;9:1018-1022.

-22 Amann K, Törnig J, Buzello M, Kuhlmann A, Gross ML, Adamczak M, Buzello M, Ritz E: Effect of antioxidant therapy with dl-alpha-tocopherol on cardiovascular structure in experimental renal failure. Kidney Int 2002;62:877-884.

23 Abd-Allah AR, Al-Majed AA, Mostafa AM, Al-Shabanah OA, Din AG, Nagi MN: Protective effect of arabic gum against cardiotoxicity induced by doxorubicin in mice: a possible mechanism of protection. J Biochem Mol Toxicol 2002;16:254-259.

24 Raggi P, Kleerekoper M: Contribution of bone and mineral abnormalities to cardiovascular disease in patients with chronic kidney disease. Clin JAm Soc Nephrol 2008;3:836-843.

-25 Gutiérrez OM, Mannstadt M, Isakova T, Rauh-Hain JA, Tamez H, Shah A, Smith K, Lee H, Thadhani R, Jüppner $\mathrm{H}$, Wolf M: Fibroblast growth factor 23 and mortality among patients undergoing hemodialysis. N Engl J Med 2008;359:584-592.

-26 Aminzadeh MA, Nicholas SB, Norris KC, Vaziri ND: Role of impaired Nrf2 activation in the pathogenesis of oxidative stress and inflammation in chronic tubulo-interstitial nephropathy. Nephrol Dial Transplant 2013;28:2038-2045.

27 Smith K, Semple D, Bhandari S, Seymour AM: Cellular basis of uraemic cardiomyopathy: a role for erythropoietin? Eur J Heart Fail 2009;11:732-738. 